\title{
Knowledge, Attitudes and Practices on Sanitation Among Women of Reproductive Age at Badbado Camp, Dharkenley District, Mogadishu-Somalia
}

\author{
Mohamed Ahmed Alasow ${ }^{1}$, Abdirizak Mohamud Yusuf ${ }^{2}$ \\ ${ }^{1}$ Faculty of Health Science, Benadir University, Mogadishu, Somalia \\ ${ }^{2}$ Department of Public Health, Head of WASH Section, Ministry of Health, Mogadishu, Somalia
}

Email address:

sadiiqnoor@gmail.com (M. A. Alasow), abdirizak992@gmail.com (A. M. Yusuf)

\section{To cite this article:}

Mohamed Ahmed Alasow, Abdirizak Mohamud Yusuf. Knowledge, Attitudes and Practices on Sanitation Among Women of Reproductive Age at Badbado Camp, Dharkenley District, Mogadishu-Somalia. Central African Journal of Public Health. Vol. 6, No. 4, 2020 , pp. $220-225$. doi: 10.11648/j.cajph.20200604.16

Received: June 16, 2020; Accepted: July 10, 2020; Published: July 23, 2020

\begin{abstract}
More than 1 billion people are lacking access to an adequate water supply and 2.6 billion people are without access to proper sanitation services. Diarrheal diseases are also the third cause responsible for increased morbidity rates in all age groups in Indonesia. In 2008 Sub-Saharan Africa the diarrhea was the leading cause of death among children under 5 years, resulting in $19 \%$ of all deaths in this age group. The Corburn and Hildebrand also found that women with reproductive age are limited or no access to toilet predominantly suffered from diarrheal diseases, a leading cause of under nutrition among women during their reproductive age. This study was to determine the level of knowledge, attitudes and practices of sanitation among women of reproductive age at Badbado Camp in Dharkenley district Mogadishu, Somalia. Methodology: The study adopted across sectional, descriptive quantitative and qualitative research approach. The target populations were women of reproductive age living in the study area. Convenient sampling technique was used to recruit the 379 study participants. Semistructured questionnaires and focus group discussion were employed in data collection. The quantitative data was analyzed using SPSS version 24 , while qualitative data was analyzed using thematic analysis. Results: It was observed that $55.8 \%$ of the participants were aware that prevention activities are crucial aspects towards diseases spread and $44 \%$ of participants were not attentive that prevention activities are important for public health measures. Similarly, 357 (93.5\%) participants reported that they normally practice hand hygiene. The $23(6.1 \%)$ participants reported that they burnt their solid waste while $2(0.5 \%)$ respondents stated that they bury their waste product. Conclusion: This study observed that almost three quarter of the participants had never got any information related to sanitation. Therefore, there is a need to build enough latrines for IDP setting in order to achieve free open defecation environment and the relevant authorities should set up waste collection stations and disposal sites in the study area to improve sanitation status.
\end{abstract}

Keywords: Sanitation, Knowledge, Practice

\section{Background}

More than 1 billion people are lacking access to an adequate water supply and 2.6 billion people are without access to proper sanitation services. Most of these people live in areas of Asia, where as much as half of the population lack of proper sanitation services, and in areas of Africa. The situation is especially alarming in rural areas, where half of the people do not have access to proper sanitation and water supply services [1]. Strunz et al, [13] identifies that women with poor sanitation facilities are more susceptible to hookworm infestation resulting in maternal anemia, which in turn is directly associated to adverse pregnancy outcomes [14, 2] the diarrhea remains second leading cause of mortality and morbidity among under-five children worldwide and the first in sub-Sahara Africa [1, 2]. Furthermore, the lives of about 800 under-five children are lost daily due to diarrhea worldwide [3]. The highest rates of child mortality are in sub-Saharan Africa and Asia is [2, 5, 6, 3]. The high mortality rates of $13.9 \%$ are still attributed to diarrheal deaths 
in Egypt among children less than five years old irrespective of the recent reduction in child mortality rates [1]. The leading cause of infant mortality and health-related expenditures has been attributed to diarrheal incidences among children in Indonesia [4] Diarrheal diseases are also the third cause responsible for increased morbidity rates in all age groups in Indonesia [4]. Similarly another study revealed that $93.1 \%$ of households disposed of food debris as waste and $77.8 \%$ disposed of plastic materials as waste. The study also showed that $61.0 \%$ of the households disposed of their waste at community bins or had waste picked up at their homes by private contractors. The remaining 39.0\% disposed of their waste in gutters, streets, holes and nearby bushes [5].

However, in humanitarian crises, WASH are amongst the principal challenge, particularly in the acute and early recovery phases, when diarrheal disease has been found to account for nearly $40 \%$ of deaths in camp residents and $80 \%$ of deaths in children under two years of age $[10-12,6]$. In sub-Saharan Africa in 2008, for example, diarrhea was the leading cause of death among children under 5 years in subSaharan Africa, resulting in $19 \%$ of all deaths in this age group [13, 7]. Nevertheless, systematic reviews suggest that improved sanitation can reduce rates of diarrheal diseases by $32 \%-37 \% \quad[14-16,7]$. However, the improvements in one or more of these three components of good health can substantially reduce the rates of morbidity and the severity of various diseases and improve the quality of life of huge numbers of people, particularly children, in developing countries $[2,3,7]$.

In Somalia, the open defecation is common practice and 28 percent of the population defecates in the open [2]. Without access to clean water, toilets and good hygiene practices, the risk of contracting easily preventable diseases, such as diarrhea, acute watery diarrhea, cholera, and respiratory infections was high. In the past 3 years, more than 900 people in Somalia, majority of them children under the age of five, have died from cholera [3, 8]. In Somalia, 37 per cent of the population practice open defecation - one of the primary causes of diarrhea. However, in Somalia's rural areas, open defecation is a common practice, leading to serious public health risks. With its local partner HEAL, UNICEF is helping villages adopt 'Community-Led Total Sanitation' to achieve the status of open defecation free [9].

\section{Methods and Materials}

\subsection{Study Design}

This research study adopted a combination of crosssectional, descriptive, quantitative and qualitative research method.

\subsection{Study Area}

The study was carried out at Badbado camp in Dharkenley district, Mogadishu-Somalia. This study was chosen because the numerous numbers of IDPs that reside there. Badbado camp is hosted largest internal displaced people from different parts in Somalia.

\subsection{Target Population}

The target populations for this study were all the women of reproductive age living in Badbado camp in Dharkenleyn district, Mogadishu-Somalia.

\subsection{Sample Size Determination}

Krejcie \& Morgan (1970) formula; $S=X^{2} N P(1-P) / d^{2}(N-$ $1)+X^{2} P(1-P)$, was used to determine the sample size. Where; $\mathrm{S}=$ required sample size, $\mathrm{X}^{2}=$ the table value of chi- square for 1 degree of freedom at desired confidence level (3.841),

$\mathrm{N}=$ study population size (29100-PESS 2014-Somalia).

$\mathrm{P}=$ population proportion (will assume to be 0.50 since this provided maximum sample size). $\mathrm{d}^{2}=$ the degree of accuracy will be expressed as a proportion 0.050 ,

$\mathrm{S}=379$ Here is mentioned $5 \%$ of non-respondent rate which is $379 \times 0.05=18$ respondents.

$\mathrm{S}=379+18=397$

$\mathrm{S}=397$

\subsection{Sampling Technique and Procedure}

The study participants were selected by using convenient sampling technique. This method was used because women in reproductive age were not well sparsely distributed, so they could not be accessed as a group to use random sampling.

\subsection{Data Collection Tool}

Quantitative data were collected using semi-structured questionnaires to obtain mother's knowledge attitude and practice towards sanitation. On the other hand, qualitative data were collected using focus group discussion.

\subsection{Data Analysis and Presentation}

Quantitative data entered and analyzed using SPSS version 24. Frequency tables and cross tabulations showing frequency counts and percentage distributions were used to present the analyzed quantitative data. The qualitative data were analyzed using thematic analysis.

\section{Results}

The findings of this study were presented as per study guiding objectives.

\subsection{Quantitative Results}

\subsubsection{Distribution of Participants by Their Sanitation Knowledge}

The distribution of participants by their knowledge as well as their sanitation practices including educational level, sanitation information, and sanitation guidance for children, awareness on disease prevention methods, prevention measures practices and respondent's awareness on disease associated with poor sanitation were analyzed and the results 
are showed in (Figure 1).
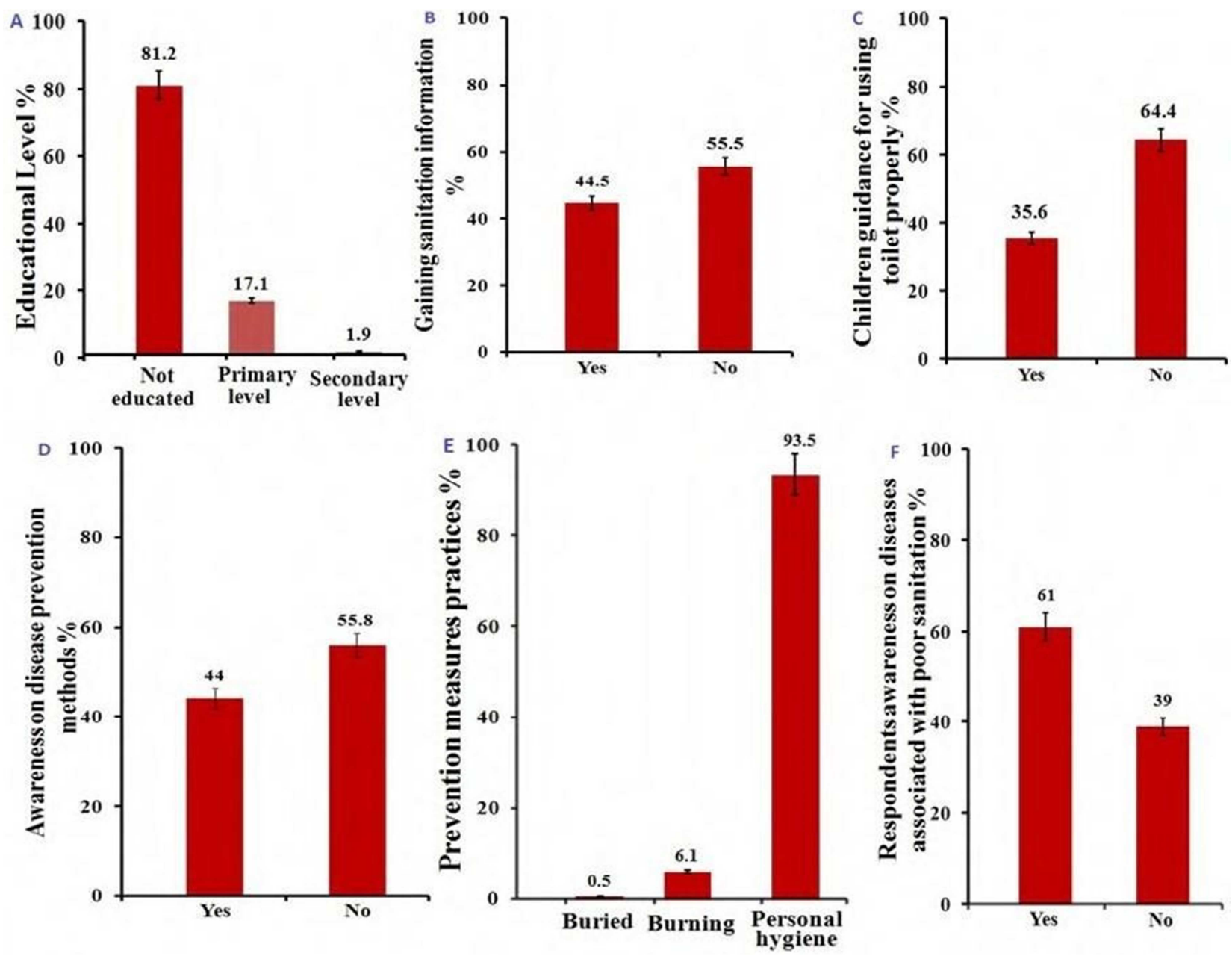

Figure 1. Distribution of participants by their sanitation knowledge, a. Educational Level, b. Gaining sanitation information, c. Children guidance for using toilet properly, $d$. Awareness on disease prevention methods, e. Prevention measures practices, $f$. Respondents awareness on diseases associated with poor sanitation. As shown in Figure 1A, the majority (81.2\%) of the participants had no educational background while (17.1\%) had attained primary education level and (1.9\%) had attained secondary level.

Therefore, it is worth noting that majority of the study participants were illiterate. The (Figure 1B), revealed that $(55.5 \%)$ were have information related sanitation while (44.5\%) were not ever got an information about the sanitation therefore this implies that around fifty percent of the respondents were not aware the importance of sanitation practices and diseases prevention related to poor sanitation.

Moreover, Figure 1C, described that the majority of the responded women $(64.4 \%)$ gave some guidance to their children to use toilet properly, (35.6\%) were not aware the role of giving guidance for using toilet to their children. so this implying that above thirty percent of the respondents were not aware the role of giving training session for their children to use toilet in a properly way.

As depicted in Figure 1D, (55.8\%) participants were aware that prevention methods are crucial aspects towards diseases spread while $44 \%$ were not aware that prevention methods are important pieces towards diseases spread. Thus, this implies that around fifty percent were not aware the prevention measures against the diseases transmission. Our study also showed that $93.5 \%$ do normally practice hand hygiene while $6.1 \%$ burn their solid wastes. Similarly $0.5 \%$ buried their waste product (Figure 1E). This implies that above ninety percent of the respondents is practicing hand hygiene which is one factor of breaking transmission of diseases among the population. Therefore, Figure 1F displayed that all of the respondents exhibited low level of awareness towards diseases association with poor sanitation

and importance to improve their knowledge to the diseases that associate with poor sanitation practices and might affect their health status. The correct answer gets: 1score and the wrong answer gets: 2 score.

The possible scores for respondents 382 to respondents' knowledge were classified into two levels. The cut-off point for 'high knowledge: greater than $70 \%$ of 382 scores and low knowledge: less than $69 \%$ of 382 scores. 
Table 1. Distribution of participants by their sanitation attitude.

\begin{tabular}{lcc}
\hline Parameter & Frequency & Percentage \\
\hline Wastes are considered one of the environmental problems that need to be solved urgently. & 55.3 \\
Agree & 211 & 15.2 \\
Disagree & 58 & 31.3 \\
strongly agree & 112 & 99.7 \\
Total & 381 & \\
Scoring result of the attitude respondents Taking local basket for shopping rather than using plastic bags is good for reducing the wastes mitigation in \\
Badbado camp & 167 & 43.8 \\
Agree & 108 & 28.3 \\
Disagree & 6 & 1.5 \\
None & 79 & 20.7 \\
Strongly agree & 22 & 5.8 \\
Undecided & 382 & 100 \\
Total & 177 & 46.4 \\
To reuse old plastic bottles as drinking water bottle can reduce waste in the Badbado camp. & 31.7 \\
Agree & 121 & 2.1 \\
Disagree & 8 & 14.4 \\
None & 55 & 5.5 \\
Strongly agree & 21 & 100.0 \\
Undecided & 382 & \\
Total & & . \\
\hline
\end{tabular}

\subsubsection{Distribution of Participants by Their Sanitation Attitudes}

As table 1 show $211(55.3 \%)$ out of 381 respondents agreed that waste is one of the environmental problems that need to be solved followed by 112 (31.3) of 381 participants who strongly agreed that wastes are environmental problems, while $58(15.2 \%)$ of 381 respondents responded disagreed so this involves the majority of respondents were aware the importance and need to improve environmental problems such as solid wastes, because majority of the respondents exhibited low level of attitude towards taking local basket for shopping rather than using plastic bags that is good for reducing the wastes mitigation in Badbado camp. The correct answer gets: 1score and the wrong answer gets: 2score.
The possible scores for respondents 382 to interviewee attitude were classified into two levels. The cut- off point for 'high attitude: greater than $70 \%$ of 382 scores and low attitude: less than $69 \%$ of 382 scores and finally 177 (46.4\%) agreed to reuse an old plastic bottles as drinking water bottle while $121(31.7 \%)$ disagreed this, other group of respondents $55(14.4 \%)$ mentioned that they strongly agree to reuse an old plastic bottles as drinking water bottles rather than disposal followed by 21 (5.5\%) of respondents undecided to mention any of the above whether agree or disagree and finally $8(2.1 \%)$ of the respondents did not mention either agree or disagree this comprises that more than thirty percent of the respondents prefer to discard bottles rather than reuse.

Table 2. Distribution of Participants by Their Sanitation Practices.

\begin{tabular}{lll}
\hline Parameter & Frequency & Percentage \\
\hline Usually separates the wastes that are normally generated from & & \\
All the time & 115 & 30.1 \\
Most of the time & 43 & 11.3 \\
Never & 128 & 33.5 \\
Rarely & 43 & 11.3 \\
Sometimes & 53 & 13.9 \\
Total & 382 & 100.0 \\
Dispose my home wastes into garbage pit near my home. & & \\
Most of The Times & 89 & 23.3 \\
Never & 100 & 26.2 \\
Rarely & 22 & 5.7 \\
Sometimes & 79 & 20.8 \\
All The Time & 92 & 24.2 \\
Total & 382 & 100.0 \\
Scoring result of the practice respondents usually burn the collected plastic wastes in order to prevent animals from eating them in Badbado camp. \\
All The times & 134 & 35.1 \\
Most of The Times & 76 & 20 \\
Never & 91 & 23.8 \\
Rarely & 22 & 5.7 \\
Sometimes & 59 & 15.5 \\
Total & 382 & 100.0 \\
\hline
\end{tabular}




\subsubsection{Distribution of Participants by Their Sanitation Practices}

As table 2 shows 128 (33.5\%) participants did not separate wastes that are normally generate from their homes were only $115(30.1 \%)$ usually separate their wastes that are Normally generate from their homes followed by 53 (13.3\%) separate their wastes that are normally generate from their homes around $43(11.3 \%)$.

Therefore, thirty percent of this study never separate the wastes that generate from their homes, but 100 (26.2\%) had not disposed their home wastes into garbage pit near their homes. Among 92 (24.2\%) were all the times disposing their home wastes into garbage pit near their homes followed by $89(23.3 \%)$ were disposing most of the times the home wastes into garbage pit near their homes because of 79 $(20.8 \%)$ were disposing sometimes their home wastes into garbage pit near their homes and only $22(5.7 \%)$ were rarely disposing their home wastes into garbage pit near their homes. The women in reproductive age who lived in Badbado camp thirty percent of them were not disposing their home wastes into garbage pit near their homes, which means their sanitation management is poor. These solid wastes accumulated near their resident areas that may be eventually lead ill-health conditions. Also, there is a low level of practices towards burning the collected plastic wastes in order to prevent animals from eating them in Badbado Camp. The correct answer gets: 1score and the wrong answer gets: 2score. The possible scores for respondents 382 to respondents' practice were classified into two levels. The cutoff point for 'high practice: greater than $70 \%$ of 382 scores and low practice: less than $69 \%$ of 382 scores.

\subsection{Qualitative Results}

In this section, qualitative data were presented thematically; five focus group discussions were conducted. The outcome of this section is presented as follows. Theme1: What do you know about sanitation? The respondents were asked their knowledge about sanitation. Half of the respondents were answered that sanitation is safe disposal of wastes while next fifty percent of the respondents replied that sanitation is to remove wastes so this seems that respondents were somehow good to understanding sanitation issues. Theme2: How did you know the information about sanitation? The respondents were asked the sources of information about the sanitation.

The majority of them mentioned that source of information about sanitation were received through radio while some of them narrated they received this information from their parents and also mentioned that information about sanitation was received from the community mobilization activities, similarly Theme3: Importance for keeping proper sanitation. The interviewees were asked about the importance of keeping sanitation and the respondents confirmed that the importance of keeping sanitation is to prevent the diarrhea diseases which are highly prevalent in Somalia and it's good to implement certain activities against the diarrheal diseases.

Therefore, Theme4: What are the diseases as a result of poor sanitation practices? This indicates that most of the women in reproductive age revealed that diseases related to poor sanitation practices were infectious diseases, although one respondent was not mentioned any disease that associate with poor sanitation practices.

\section{Discussion}

This study indicating that the majority of the participants (81.2\%) had no educational background, while $(17.1 \%)$ had attained primary education level, and only (1.9\%) had attained secondary level. Therefore, it is worth noting that majority of the study participants were illiterate. However, another study in Badbado camp where reflected the most of disposal of solid wastes are a major problem in urban cities. The cities in East and North Africa as well as most developing countries are also facing the similar problems related to safe sanitation and waste garbage disposal. The main reason of these problems is attributed to the poor economy of these areas which accounts for the low achievement in solid waste management [14].

The caretakers $(66.1 \%)$ had illiterate, were one third was $(33.9 \%)$ and the $(23.6 \%)$ of the caretakers were Quran level of education, while few (1.8\%) were secondary level [10]. Our study also showed that $93.5 \%$ do normally practice hand hygiene although $6.1 \%$ burn their solid wastes.

Only $0.5 \%$ buried their waste product, therefore this result of ninety percent of the respondents is practicing hand hygiene, which is one factor of breaking transmission of diseases among the population another Studies offer convincing and important evidence that providing a conveniently located hand hygiene sink in each client/patient/resident room reduces HAIs rates. 150 [11]. The 381 respondents agreed that waste is one of the environmental problems, that needs to be solved followed by $112(31.3 \%)$ of 381 participants who strongly agreed that wastes are environmental hazard, while $58(15.2 \%)$ of 381 respondents responded disagreed so this result. Another study, reflected of solid wastes to surface waters may also result in acute and chronic human health problems. The primary route to exposure is through drinking water. However, the consumption of fish and other aquatic organisms that accumulate chemicals is also a concern, since human health effects may occur at lower levels of bioaccumulation relative to those that effect indigenous aquatic organisms (18) [12]. This study revealed that $(55.5 \%)$ were having information related sanitation and (44.5\%) were not ever got information about the sanitation. Therefore this implies that around fifty percent of the respondents were not aware the importance of sanitation practices as too of diseases prevention associated with poor sanitation. On the other hand, another study indicates that due to unplanned communities and developments in major cities, an environmental and sanitary condition is becoming very complex [13]. Due to a lack of awareness and low income sources, dwellers are forced to live with unhealthy and unhygienic conditions [13] however, 
the participants $100(26.2 \%)$ never disposed their home wastes into garbage pit near their homes. while 92 (24.2\%) were all the times disposing their home wastes into garbage pit near their homes followed by $89(23.3 \%)$ were disposing most of the times the home wastes into garbage pit near their homes due to $79(20.8 \%)$ were disposing sometimes their home wastes into garbage pit near their homes and 22 (5.7\%) were rarely disposing their home wastes into garbage pit near homes. This entails that thirty percent of women in reproductive age who lived in Badbado camp were never disposing their home wastes into garbage pit near their homes. Another study shows that several studies showed that collection, storage, transportation and final.

\section{Conclusion and Recommendation}

It is necessary to establish community health mobilization in order to achieve better health living environment. There is need to distribute sanitation kit items to the community who are living at Badbado camp in Dharkenleyn district to eliminate the diseases that are associated with poor sanitation. It is necessary to build enough latrines camps in order to achieve open defecation free. It is very important to build waste collection stations and disposal sites at Badbado Camp in Dharkenleyn District for proper sanitation.

\section{References}

[1] Huuhtanen, S. and Laukkanen, A. A guide to sanitation and hygiene for those working in developing countries, Tampere polytechnic. 2006. ISBN952-5264-49-1.

[2] Saleem, M, Burdett, T, Heaslip, V,. Health and social impacts of open defecation on women: a systematic review, 2019, https://doi.org/10.1186/s12889-019-6423-z.

[3] Mshida H, Kassim N, Kimanya M, Mpolya E, Influence of Water, Sanitation, and Hygiene Practices on Common Infections among Under-Five Children in Longido and Monduli Districts of Arusha, Tanzania, JEPH (2017) Article ID 92351688 pages | https://doi.org/10.1155/2017/9235168.

[4] Joshi A and Amadi C. Impact of Water, Sanitation, and Hygiene Interventions on Improving Health Outcomes among School Children. (2013). Journal of Environmental and Public Health Volume 2013. Article ID 984626-10 pages. http://dx.doi.org/10.1155/2013/984626.
[5] Yoada, R, Chirawurah, D, Adongo P, Domestic waste disposal practice and perceptions of private sector waste management in urban Accra,(2014), BMC Public Health, doi: 10.1186/1471-2458-14-697.

https://www.ncbi.nlm.nih.gov/pmc/articles/PMC4226987/.

[6] Ramesh A, Blanchet K, Ensink JHJ, Roberts B Evidence on the Effectiveness of Water, Sanitation, and Hygiene (WASH) Interventions on Health Outcomes in Humanitarian Crises: A Systematic Review, PLOSONE. 2015.|DOI: 10.1371/journal.pone.0124688.https://journals.plos.org/ploson e/article?id=10.1371/journal.pone. 0124688 .

[7] Mara D, Lane J, Scott B, Trouba D. Sanitation and Health. 2010. PLoS Med 7 (11): e1000363. doi: 10.1371/journal.pmed.1000363.

[8] UNICEF -Somalia. Water, sanitation and hygiene. Every child has the right to water, sanitation and a safe and clean community Unicef.org/Somalia (2019). https://www.unicef.org/somalia/water-sanitation-and-hygiene.

[9] UNICEF- Somalia. In Somalia, villages work together to end open defecation Unicef-somalia (2016). https://www.unicef.org/wash/somalia_94427.html.

[10] Yusuf A. M. Omer A. Yasin S. Had H. Ali M. Prevalence and factors associated with diarrhea among children aged 0-59 months in Badbaado Camp in Mogadishu, Somalia 2018? Researchjournali's Journal of Public Health. 2018. Vol. 4|No. 11.

[11] PIDAC. Best Practices for Hand Hygiene in All Health Care Settings, 4th edition (2014). Public Health Ontario. https://www.publichealthontario.ca/-

/media/documents/B/2014/bp-hand-hygiene.pdf?la=en.

[12] Qrenawi L. Environmental and Health Risk Assessment of AlAkaider Landfill. (2006). file://C:/Users/pc/Downloads/LuayThesis\%20(1).pdf.

[13] Ejaz N., Akhtar N. Nisar H. Naeem U. Environmental impacts of improper solid waste management in developing countries: a case study of Rawalpindi City. WIT Transactions on Ecology and the Environment. 2010. Vol 142, Page 380. Doi: 10.2495/SW10035.

[14] I. Hussein. Shafy, Abdel. Mansou, Solid waste issue: Sources, composition, disposal, recycling, and valorization. M. Egyptian Journal of Petroleum. 2018.1275-1290, https://doi.org/10.1016/j.ejpe.2018.07.003. 